Proc. Estonian Acad. Sci. Biol. Ecol., 2002, 51, 2, 103-123

\title{
Phylogenetic analysis of cetrarioid lichens with globose ascospores
}

\author{
Andres Saag ${ }^{\mathrm{a}}$, Tiina Randlane ${ }^{\mathrm{a}}$, Arne Thell ${ }^{\mathrm{b}}$, and Walter Obermayer ${ }^{\mathrm{c}}$ \\ a Institute of Botany and Ecology, University of Tartu, Lai 38, 51005 Tartu, Estonia; asaag@ut.ee; \\ randlane@ut.ee \\ b Department of General Botany and Botanical Garden, University of Hamburg, Ohnhorstrasse 18, \\ 22609 Hamburg, Germany; fb0a082@ botanik.uni-hamburg.de \\ c Institute of Botany, Karl-Franzens-Universität Graz, Holteigasse 6, A-8010 Graz, Austria; \\ walter.obermayer@uni-graz.at
}

Received 27 September 2001, in revised form 13 November 2001

\begin{abstract}
The group of cetrarioid lichens with globose ascospores includes 37 species from eight genera. Phylogenetic analysis, using program PAUP 3.1.1, was carried out on two different data sets - morphological (incl. anatomical and chemical) and molecular characters (ITS1, 5.8S, and ITS2 rDNA sequences) - to verify the correspondence of current taxonomy to the probable evolution of the taxa involved. The paraphyletic nature of the genus Allocetraria in regard to two species of Dactylina is assumed based on morphological characters. Still, the separation of these genera is strongly supported by the analysis of molecular data. The genus Tuckermannopsis (11 species) is paraphyletic in regard to Esslingeriana idahoensis and "Nephromopsis" weii. Monophyletic origin can be declared only for the Tuckermannopsis ciliaris group. The analysis based on molecular characters exhibits the monophyletic origin of the group including three Tuckneraria species. Monophyly of the genus Ahtiana was not supported by the analysis of morphological data.
\end{abstract}

Key words: cetrarioid lichens, Parmeliaceae, Ahtiana, Allocetraria, Dactylina, Esslingeriana, "Nephromopsis" weii, Tuckneraria, Tuckermannopsis, Vulpicida, ITS rDNA sequences, cladistic analysis.

\section{INTRODUCTION}

The group of cetrarioid lichens, comprising 23 genera and about 140 species within the family Parmeliaceae (Randlane et al., 1997; Randlane \& Saag, 2000), is a very diverse and definitely polyphyletic assemblage. Phylogenetic affinities between several cetrarioid genera are rather obscure. The first review of the phylogeny of a major part of cetrarioid lichens was presented by Kärnefelt et al. (1992) where cladistic analyses were carried out for 50 species as terminal taxa. This analysis was mainly based on anatomical characters of reproductive structures 
that had been revised originally. As a result, the analysed taxa were grouped into three separate aggregates, described by important anatomical characters: taxa with an "apical ring structure", taxa with "uniseriate asci", and taxa with "broadly clavate asci". Another cladistic analysis of the same group of lichens was carried out independently (Saag \& Randlane, 1995). In order to evaluate the systematic arrangement of the taxa 83 species were chosen for analysis from among approximately 120 cetrarioid lichen species known at the time. Morphological and chemical characters (character states) were identified or verified by the authors, while descriptions of anatomical characters were based mainly on literature data. Most of the species were grouped in a way that showed considerable similarity to the main three assemblages pointed out by Kärnefelt et al. (1992), while the taxonomy of several species of the genera Nephromopsis, Cetreliopsis, and Cetrelia remained unresolved. Similar results are particularly noteworthy because of the fact that the two analyses were based on different data matrices with emphasis on anatomical characters in one (Kärnefelt et al., 1992) and on morphological and chemical characters in the other (Saag \& Randlane, 1995) study.

A few years later the three aggregates within the group of cetrarioid lichens referred to above were characterized in precise anatomical detail (Thell, 1996). The aggregates were called "evolutionary lines" although additional cladistic analyses were not performed. The first line, proposed as monophyletic, included species from seven genera: Arctocetraria, Cetraria s. str., Cetreliopsis, Coelopogon, Flavocetraria, Masonhalea, and Nephromopsis. They all were characterized by ellipsoid ascospores in narrowly clavate asci with a small axial body. Furthermore, an amyloid ring structure in tholus was pointed out as a significant character present in Cetraria, Flavocetraria, and occasionally in Nephromopsis. The second evolutionary line comprised six genera: Ahtiana, Allocetraria, Dactylina, Esslingeriana, Tuckneraria, and Tuckermannopsis. The species of these genera were anatomically characterized by subglobose to globose ascospores borne in narrowly clavate asci (earlier called "uniseriate asci"). The assumption of the monophyletic origin of this line was based on two earlier cladistic analyses of various species of cetrarioid lichens (Kärnefelt et al., 1992; Saag \& Randlane, 1995). In both analyses, the clade comprising species with globose ascospores in narrowly clavate asci was clearly separated. The third line was represented by species of several genera and informal groups: Asahinea, the "Cetraria" fendleri group, Cetrariella, Cetrelia, Cornicularia, Kaernefeltia, the Melanelia commixta group, Nimisia, Parmelaria, Platismatia, and Vulpicida. Ellipsoid ascospores in broadly clavate asci with a broad axial body were pointed out as characteristic of these species. This heterogeneous assemblage is presumably paraphyletic as a number of parmelioid lichens most likely also belong here.

In further studies (Crespo \& Cubero, 1998; Mattsson \& Wedin, 1998; Thell, 1998, 1999; Thell \& Miao, 1998; Thell et al., 1998, 2000; Crespo et al., 1999; Wedin et al., 1999; Kärnefelt \& Thell, 2000, 2001), accumulation of molecular data has been the primary source which would allow improvement of investigations of phylogeny within the Parmeliaceae. Different regions of nuclear 
rDNA (ITS, 5.8S, group I intron) have been studied in several taxa (including cetrarioid genera, e.g., Ahtiana, Cetraria, Cetrariella, Dactylina, Platismatia, Tuckermannopsis, Vulpicida). It has been presumed that there are uncertainties in the reliability of some of the sexual reproductive structures used to identify monophyletic groups in cetrarioid lichens (Mattsson \& Wedin, 1998). Still, so far no serious phylogenetic hypotheses have been proposed based on sequences and comprising a large number of the members of this large family.

The group of cetrarioid species with globose ascospores is discussed here in more detail. This group covers the second evolutionary line referred to above in full and, in addition, includes some taxa (e.g., species of Vulpicida) from the third evolutionary line. Five of the seven genera, Ahtiana, Allocetraria, Dactylina, Tuckneraria, and Vulpicida, have recently been thoroughly revised (Mattsson, 1993; Randlane et al., 1994; Thell et al., 1995a, b; Kärnefelt \& Thell, 1996). The sole species of the monotypic genus Esslingeriana was described in detail by Esslinger (1971). The genus Tuckermannopsis in the strict sense has also recently been circumscribed, both on morphological and molecular data (Kärnefelt \& Thell, 2001). Another newly described species - "Nephromopsis" weii X. Q. Gao $\& \mathrm{~L}$. H. Chen - has also been included in the ingroup because its ascospores are clearly globose (Chen \& Gao, 2001).

Nevertheless, the data characterizing all these taxa are scattered in many papers and evolutionary affinities between the species included have not been evaluated.

\section{MATERIAL AND METHODS}

\section{Material}

Herbarium material from B, DUKE, FH, GZU, H, KW, LD, LE, M, MB, PC, S, TAIM, TNS, TU, UPS, US was used for this study. Morphology of the herbarium specimens was examined using stereomicroscopes Technival 2 and Olympus SZ40. The anatomical methods and equipment used are described in detail in Thell et al. (1995a). Secondary chemistry was investigated according to standardized methods of TLC (Culberson, 1972; Culberson et al., 1981).

\section{Molecular methods}

Sequences from the internal transcribed spacer 1 (ITS1), the 5.8S rDNA gene, and the internal transcribed spacer 2 (ITS2) of the nuclear rDNA gene were used for the molecular analysis.

\section{Extraction}

Fresh material, at least not older than three years, was used for extracting total DNA. Minute thallus fragments, $1-3 \mathrm{~mm}$ in diameter, were used for the extractions, which were performed according to the manufacturer's protocol with 
one exception: the samples were carefully ground in $50 \mu \mathrm{L}$ of the lysis buffer. As the next step, the rest of the lysis buffer, $350 \mu \mathrm{L}$, together with $10 \mu \mathrm{L}$ of RNase A $(100 \mathrm{mg} / \mathrm{mL})$, was added to each sample. Finally, the extracted DNA was eluted in $100 \mu \mathrm{L}$ of the elution buffer delivered with the NucleoSpin Plant Extraction Kit from MACHEREY-NAGEL GmbH \& Co, which was successfully used for the amplification without further dilutions.

\section{Amplification}

The ITS regions and the 5.8S gene of the nuclear rDNA were amplified using a Perkin-Elmer Gene Amp PCR System 9700 thermal cycler. Ready-To-Go PCR beads in $0.2 \mathrm{~mL}$ tubes (Pharmacia Biotech Inc.) were used in amplification. The primers ITS1F and ITS4 were used for amplifying the ITS regions. A solution containing $11.8 \mu \mathrm{L}$ of distilled water, $0.35 \mu \mathrm{L}$ each of the primers at $16 \mathrm{pmol}$ concentration, and $12.5 \mu \mathrm{L}$ of DNA was added to the PCR beads (reaction size ca. $25 \mu \mathrm{L}$ for $0.2 \mathrm{~mL}$ tubes). Following an initial 2-min template denaturing at $95^{\circ} \mathrm{C}$, a 30 -cycle schedule was performed. The following cycling profile was used: denaturation at $95^{\circ} \mathrm{C}$ for $1 \mathrm{~min}$, annealing at $60^{\circ} \mathrm{C}$ for $1 \mathrm{~min}$, and extension at $72^{\circ} \mathrm{C}$ for $1 \mathrm{~min}$. The PCR products were cleaned with QIAquick PCR purification kit (QIAGEN) and diluted in $50 \mu \mathrm{L}$ of the elution buffer provided in the kit.

\section{Sequencing and alignment}

The sequencing reactions were prepared according to the protocol provided in the BigDye Terminator Cycle Sequencing Ready Reaction Kit (PE Biosystems). In a $20 \mu \mathrm{L}$ reaction, $8 \mu \mathrm{L}$ of BigDye and buffer $(2.3 \mu \mathrm{L}$ and $5.7 \mu \mathrm{L}$, respectively), $2 \mu \mathrm{L}$ of the primers at 5 pmol concentration, $4-6 \mu \mathrm{L}$ of the purified PCR product, and $4-6 \mu \mathrm{L}$ of distilled water were mixed. A 25 -cycle schedule including denaturation at $96^{\circ} \mathrm{C}$ for $30 \mathrm{~s}$, annealing at $50^{\circ} \mathrm{C}$ for $15 \mathrm{~s}$, and extension at $60^{\circ} \mathrm{C}$ for $4 \mathrm{~min}$ was performed. The primers ITS1LM and ITS4 were used for the sequencing reactions above (Table 1). ITS1LM was here selected instead of ITS1F to avoid possible introns at the $3^{\prime}$ end of the $18 \mathrm{~S}$ gene. The sequencing was performed with an automatic sequencer ABI Prism 377 (PE Biosystems).

The sequences were aligned with SeqApp/CAP2 (Huang, 1992; Gilbert, 1993), optimized by hand and transferred to PAUP 3.1.1 (Swofford, 1993).

Table 1. Primers used in the molecular analysis (IST1LM was used for the sequencing reaction only)

\begin{tabular}{l|l|l}
\hline \multicolumn{1}{c|}{ Primer } & \multicolumn{1}{c}{ Sequence } & \multicolumn{1}{c}{ Reference } \\
\hline ITS1F* & CTTGGTCATTTAGAGGAAGTAA & Gardes \& Bruns, 1993 \\
ITS1LM & GAACCTGCGGAAGGATCATT & Myllys et al., 1999 \\
ITS4 & TCCTCCGCTTATTGATATGC & White et al., 1990
\end{tabular}

* Fungal specific. 


\section{Phylogenetic analyses}

The computer program for phylogenetic analysis using maximum parsimony as the optimization criterion, PAUP 3.1.1 (Swofford, 1993), was applied. Cladistic analyses were run on a Power Macintosh G3. The following heuristic search settings were applied: character-state optimization - DELTRAN; MULPARS; addition sequence - random; 1 tree held at each step during stepwise addition; tree-bisection-recollection (TBR) branch-swapping performed; all multi-state characters treated as unordered; when taxa are coded as having multiple states, multiple state interpreted as uncertainty; characters weighted equally. The same settings (except the last) were used in the successive approximations character weighting method. Tree support was investigated using Bremer support and bootstrap analysis. A hundred (for morphological data) or a thousand (for molecular data) bootstrap replicates were performed, with random addition sequence.

Separate analyses were carried out on two different data sets: morphological (incl. anatomical and chemical) data only and molecular data (ITS1, 5.8S, ITS2 sequences of rDNA gene) only.

\section{Data}

\section{Taxa analysed}

A total of 41 cetrarioid species were included in the morphological data matrix. The following 31 species from the above-listed genera were applied as terminal taxa of the ingroup: Ahtiana aurescens (Tuck.) Randlane \& A. Thell, A. pallidula (Tuck. ex Riddle) Goward \& A. Thell, A. sphaerosporella (Müll. Arg.) Goward, Allocetraria ambigua (Bab.) Kurok. \& M. J. Lai, A. endochrysea (Lynge) Kärnefelt \& A. Thell, A.flavonigrescens A. Thell \& Randlane, A. globulans (Nyl.) A. Thell \& Randlane, A. madreporiformis (Ach.) Kärnefelt \& A. Thell, A. oakesiana (Tuck.) Randlane \& A. Thell, A. sinensis X. Q. Gao, A. stracheyi (Bab.) Kurok. \& M. J. Lai, Dactylina arctica (Richardson) Tuck., D. ramulosa (Hook.) Tuck., Esslingeriana idahoensis (Essl.) Hale \& M. J. Lai, "Nephromopsis" weii X. Q. Gao \& L. H. Chen, Tuckermannopsis americana (Spreng.) Hale, T. chlorophylla (Willd.) Hale, T. ciliaris (Ach.) Gyeln., T. gilva (Asahina) M. J. Lai, T. inermis (Nyl.) Kärnefelt, T. microphyllica (W. L. Culb. \& C. F. Culb.) M. J. Lai, T. orbata (Nyl.) M. J. Lai, T. platyphylla (Tuck.) Hale, T. platyphylloides (Asahina) M. J. Lai, T. subalpina (Imshaug) Kärnefelt, T. ulophylloides (Asahina) M. J. Lai, Tuckneraria ahtii Randlane \& Saag, T. laureri (Kremp.) Randlane \& A. Thell, T. laxa (Zahlbr.) Randlane \& A. Thell, T. pseudocomplicata (Asahina) Randlane \& Saag, and T. togashii (Asahina) Randlane \& A. Thell.

The cladograms were rooted using the genus Vulpicida (with $V$. canadensis (Räsänen) J.-E. Mattsson \& M. J. Lai, V. juniperina (L.) J.-E. Mattsson \& M. J. Lai, V. pinastri (Scop.) J.-E. Mattsson \& M. J. Lai, V. tilesii (Ach.) 
J.-E. Mattsson \& M. J. Lai, V. tubulosa (Schaer.) J.-E. Mattsson \& M. J. Lai, and V. viridis (Schwein.) J.-E. Mattsson \& M. J. Lai in the outgroup). At first, the six species of Vulpicida were also included in the ingroup because the ascospores are mainly subglobose in this genus. Then several outgroup candidates, e.g. "Cetraria" fendleri (Nyl.) Tuck., Cetraria islandica (L.) Ach., Flavocetraria cucullata (Bellardi) Kärnefelt \& A. Thell, and Melanelia hepatizon (Ach.) A. Thell, from different cetrarioid genera were tested. The analyses where "Cetraria" fendleri, Cetraria islandica, and Flavocetraria cucullata were separately used as outgroups resulted in much less resolution than the analysis with Melanelia hepatizon as an outgroup. On the strict consensus tree of that analysis, the species of Vulpicida appeared as a sister group to the rest of the species included. Vulpicida was therefore selected as the outgroup for further analyses. The genus Vulpicida is anatomically characterized by almost subglobose ascospores and broadly clavate asci of Melanelia-form with a large axial body (Thell et al., 1995b) while the taxa of the ingroup have globose to subglobose ascospores borne in narrowly clavate asci with a large axial body (Tuckermannopsis-form according to Thell et al., 1995b).

\section{Morphological characters}

All in all, 37 morphological characters were used for the analyses (Table 2). They belong to three main groups, describing thallus morphology and general organization (characters 1-16), reproductive structures and their anatomy (17-27), and secondary chemistry (28-37). Lichen substances were not treated independently from each other but grouped into biochemically related sets, as suggested in a previous study (Saag \& Randlane, 1995).

Character states were coded as $0,1,2,3$, and 4 . The characters and character states were as follows.

1. Form of thallus: adnate (0), ascending (1)

2. Symmetry of thallus: dorsiventral (0), radial-symmetrical (1)

3. Interior of thallus: of densely arranged hyphae (0), of loosely arranged hyphae (1), becoming hollow (2)

4. Form of lobes: length of lobes $\sim$ width of lobes $(0)$, lobes longer than wide (1)

5. Width of lobes: up to $3 \mathrm{~mm}(0)$, up to $6 \mathrm{~mm}$ (1), up to $12 \mathrm{~mm} \mathrm{(2)}$

6. Colour of upper surface: yellow (0), brown (1), grey (2)

7. Colour of lower surface: whitish (0), yellow (1), brown (2), black (3)

8. Pseudocyphellae on upper side: absent (0), present (1)

9. Pseudocyphellae on lower side: absent (0), present (1)

10. Form of pseudocyphellae on lower side: spots (0), lines (1)

11. Cilia: absent (0), present (1)

12. Rhizines: absent (0), present (1)

13. Soredia: absent (0), present (1) 
14. Isidia: absent (0), present (1)

15. Structure of the cortex (orientation of hyphae): both cortices paraplectenchymatous, i.e. hyphae randomly oriented (0); upper cortex palisade plectenchymatous, i.e. hyphae anticlinally oriented (1); both cortices palisade (2); both cortices prosoplectenchymatous, i.e. hyphae parallel to cortex (3)

16. Cell wall thickness (compared to cell lumina): leptodermatous, i.e. hyphae with thin cell walls and lumen is clearly larger in diameter than the adjacent cell walls (0); pachydermatous, i.e. hyphae with thick cell walls and lumen is smaller in diameter than the adjacent cell walls (1)

17. Position of apothecia: marginal only (0), marginal to submarginal (1), laminal (2), terminal (3)

18. Ascus shape: broadly clavate (0), narrowly clavate (1)

19. Ascus form according to Thell et al. (1995b): Melanelia-form (0), Tuckermannopsis-form (1), Cetraria-type (2)

20. Tholus: small (0), large (1)

21. Axial body: medium, $3-5 \mu \mathrm{m}(0)$; broad, $>5 \mu \mathrm{m}(1)$; narrow, $>1$ and $<3 \mu \mathrm{m}(2)$, very narrow, $\leq 1 \mu \mathrm{m}(3)$

22. Shape of ascospores: globose (0), subglobose (1), broadly ellipsoid (2), ellipsoid (3)

23. Length or diameter of ascospores: short, $\leq 6 \mu \mathrm{m}(0)$; long, $>6 \mu \mathrm{m}$ (1)

24. Position of pycnidia: marginal only (0), marginal and laminal (1), laminal only (2)

25. Emergence of pycnidia: emergent (0), immersed (1)

26. Shape of pycnoconidia: bacillariform (0), oblong citriform (1), dumb-bell shaped incl. disc-bar shaped (2), filiform (3), sublageniform (4)

27. Length of pycnoconidia: short, $<7 \mu \mathrm{m}(0)$; medium, 7-10 $\mu \mathrm{m}$ (1); long, $>10 \mu \mathrm{m}(2)$

28. Usnic acid: absent (0), present (1)

29. Atranorin: absent (0), present (1)

30. Fatty acids: absent $(0)$, present $(1)$

31. Substance of fatty acids: lichesterinic-protolichesterinic type acids (0), caperatic acid (1), rangiformic acid (2), unidentified (3)

32. Secalonic acids: absent (0), present (1)

33. Orcinol depsides and depsidones: absent (0), present (1)

34. Substance of orcinol depsides and depsidones: alectoronic and collatolic acids (0), physodic acid (1), olivetoric acid (2), microphyllinic acid (3), gyrophoric acid (4)

35. B-Orcinol depsidones: absent (0), present (1)

36. Substance of $\beta$-orcinol depsidones: fumarprotocetraric acid ( 0$)$, physodalic acid (1)

37. Pulvinic acid derivates: absent (0), present (1) 
Table 2. Morphological data matrix

\begin{tabular}{|c|c|c|c|c|c|c|c|c|c|c|c|c|c|c|c|c|c|c|}
\hline \multirow{2}{*}{ axon } & \multicolumn{18}{|c|}{ Character number and states } \\
\hline & 1 & 2 & 3 & 4 & 5 & 6 & 7 & 8 & 9 & 10 & 11 & 12 & 13 & 14 & 15 & 16 & 17 & 18 \\
\hline Ahtiana aurescens & 0 & 0 & 0 & 0 & 0 & 0 & 0 & 0 & 0 & - & 1 & 1 & 0 & $0 \& 1$ & 0 & 1 & 1 & 1 \\
\hline A. pallidula & 1 & 0 & 0 & 0 & 2 & 0 & 1 & 0 & 0 & - & 0 & 1 & 0 & 0 & 0 & 1 & 1 & 1 \\
\hline A. sphaerospo & 0 & 0 & 0 & 0 & 0 & 0 & 0 & 0 & 0 & - & 0 & 1 & 0 & 0 & 0 & 0 & 2 & \\
\hline Allocetraria ambigua & 1 & 0 & 0 & 1 & 1 & 0 & 1 & 0 & 1 & 0 & 0 & 1 & 0 & 0 & 2 & 1 & 0 & 1 \\
\hline A. endochrysea & 1 & 1 & 1 & 1 & 0 & 0 & 1 & 0 & 0 & - & 0 & 1 & 0 & c & 2 & 0 & $?$ & $?$ \\
\hline A. flavoni & 1 & 0 & 0 & 1 & 0 & 0 & $2 / 3$ & 0 & 0 & - & 0 & 1 & 0 & 0 & 2 & 0 & 1 & 1 \\
\hline A. globular & 0 & 0 & 0 & 0 & 1 & $0 / 1$ & 2 & 0 & 0 & - & 0 & 1 & 0 & 0 & 2 & 0 & 1 & 1 \\
\hline A. $n$ & 1 & $0 \& 1$ & 1 & 1 & 0 & 0 & 1 & 0 & 0 & - & 0 & 0 & 0 & 0 & 2 & 0 & 3 & 1 \\
\hline A. oakesic & 0 & 0 & 0 & 0 & 1 & 0 & 2 & 0 & 0 & - & 0 & 1 & 1 & 0 & $0 / 2$ & 0 & 1 & 1 \\
\hline A. sinensis & 1 & 0 & 0 & 1 & 0 & 0 & 2 & 0 & 1 & 1 & 0 & 0 & 0 & 0 & 2 & 0 & $?$ & ? \\
\hline A. $s$ & 1 & $0 \& 1$ & 0 & 1 & 1 & 0 & $1 / 2$ & 0 & 1 & 0 & 0 & 1 & 0 & 0 & 2 & 1 & 0 & 1 \\
\hline Dactylina & 1 & 1 & 2 & 1 & 2 & $0 / 1$ & $1 / 2$ & 0 & 0 & - & 0 & 0 & 0 & 0 & 2 & 0 & 3 & 1 \\
\hline D. $r a$ & 1 & 1 & 2 & 1 & 0 & 1 & 1 & 0 & 0 & - & 0 & 0 & 0 & 0 & $2 \& 3$ & 1 & 3 & 1 \\
\hline Essl & 0 & 0 & 0 & $0 \& 1$ & 1 & 2 & 3 & 0 & 0 & - & 0 & 1 & 0 & 0 & 0 & 0 & 1 & 1 \\
\hline idah & & & & & & & & & & & & & & & & & & \\
\hline "Nepht & 1 & 0 & 0 & 0 & 2 & 1 & 2 & 1 & 1 & $?$ & 0 & 1 & 0 & 0 & 0 & $?$ & 0 & 1 \\
\hline & 0 & 0 & 0 & 0 & 1 & 1 & 2 & 0 & 0 & - & 1 & 1 & 0 & 0 & 0 & 0 & 1 & 1 \\
\hline T. chlorophylla & 0 & 0 & 0 & 0 & 1 & 1 & 2 & 0 & 0 & - & 0 & 1 & 1 & 0 & 0 & 0 & 0 & 1 \\
\hline & 0 & 0 & 0 & 0 & 1 & 1 & 2 & 0 & 0 & - & 1 & 1 & 0 & 0 & 0 & 0 & 1 & 1 \\
\hline T. gilva & 0 & 0 & 0 & 0 & 1 & 1 & 2 & 0 & 0 & - & 1 & 1 & 1 & c & 0 & 0 & $?$ & ? \\
\hline T. it & $0 \& 1$ & 0 & 0 & 0 & 0 & 1 & 0 & 0 & 1 & 1 & 0 & 1 & 0 & 0 & 0 & 1 & 1 & 1 \\
\hline T. microp & 0 & 0 & 0 & 0 & 1 & 1 & 2 & ( & 0 & - & 0 & 1 & 0 & 0 & 0 & 1 & 1 & 1 \\
\hline T. orbata & 0 & 0 & 0 & 0 & 1 & 1 & 2 & 0 & 0 & - & 1 & 1 & 0 & 0 & 0 & 0 & 0 & 1 \\
\hline T. $p$ & $0 \& 1$ & 0 & 0 & 0 & 2 & 1 & 2 & & 0 & - & 0 & 1 & 0 & 1 & 0 & 1 & 1 & 1 \\
\hline T. platyph & 0 & 0 & 0 & 0 & 2 & 1 & 2 & 0 & 0 & - & 0 & 1 & 0 & 0 & 0 & 0 & 0 & ? \\
\hline T. subalpina & 1 & 0 & 0 & 1 & 1 & 1 & 2 & 0 & 1 & 1 & 0 & 0 & 0 & 0 & 0 & 0 & 0 & 1 \\
\hline T. $u$ & 0 & 0 & 0 & 0 & 1 & 1 & $1 / 2$ & 0 & 0 & - & 0 & 1 & 1 & 1 & 1 & 0 & $?$ & ? \\
\hline Tuckneraria ahtii & 1 & 0 & 0 & 1 & 2 & 0 & $2 / 3$ & 0 & 1 & 0 & 1 & 1 & 0 & 0 & 0 & 1 & 0 & 1 \\
\hline & $0 \& 1$ & 0 & 0 & 0 & 1 & 0 & $0 / 2$ & 0 & 1 & 0 & 1 & 1 & 1 & 0 & 0 & 1 & 0 & 1 \\
\hline T. laxa & 0 & 0 & 0 & 1 & 0 & 0 & 1 & 0 & 1 & 0 & 1 & 1 & 0 & 0 & 0 & 1 & 0 & 1 \\
\hline T. pseudoc & 1 & 0 & 0 & 0 & 2 & 0 & $0 / 2$ & 0 & 1 & 0 & 1 & 1 & 0 & 0 & 0 & 1 & 0 & 1 \\
\hline & 0 & 0 & 0 & 0 & 1 & 0 & $1 / 2$ & 0 & 1 & 0 & 1 & 1 & 0 & 1 & 0 & 1 & 0 & 1 \\
\hline Vulpicida c & 1 & 0 & 0 & 0 & 2 & 0 & 1 & 0 & 0 & - & 0 & $0 \& 1$ & 0 & 0 & 0 & 1 & 2 & 0 \\
\hline$V$. juniperina & $0 \& 1$ & 0 & 0 & 0 & 1 & 0 & 1 & 0 & 0 & - & 0 & 1 & 0 & 0 & 0 & 1 & 1 & 0 \\
\hline$V \cdot$ pinastr & 0 & 0 & 0 & 0 & 1 & 0 & 1 & 0 & 0 & - & 0 & 1 & 1 & 0 & 0 & 1 & 1 & 0 \\
\hline V. tilesii & 1 & 0 & 0 & 0 & 0 & 0 & 1 & 0 & 0 & - & 0 & 0 & 0 & 0 & 0 & 1 & 1 & 0 \\
\hline & 1 & $0 \& 1$ & 0 & 1 & 0 & 0 & 1 & 0 & 0 & - & 0 & 0 & 0 & 0 & 0 & 1 & 1 & 0 \\
\hline V. viridis & 0 & 0 & 0 & 0 & 1 & 0 & 1 & 0 & 0 & - & 0 & 1 & 0 & 0 & 0 & 1 & 2 & 0 \\
\hline "Cetraria" fendleri & 0 & 0 & 0 & 0 & 0 & 1 & 0 & 0 & 0 & - & 0 & 1 & 0 & 0 & 0 & 1 & $1 \& 2$ & 0 \\
\hline & 1 & 0 & 0 & 1 & 2 & 1 & 2 & & 1 & 0 & 0 & 0 & 0 & 0 & $0 \& 3$ & 1 & 3 & \\
\hline Flavocetra & 1 & 0 & 0 & 1 & 1 & 0 & 1 & 0 & 1 & 0 & 0 & 0 & 0 & 0 & $0 / 2$ & 1 & 3 & \\
\hline Melanelia hepatizon & 0 & 0 & 0 & 0 & 0 & 1 & 3 & 1 & 0 & - & 0 & 1 & 0 & 0 & 0 & & $1 \& 2$ & \\
\hline
\end{tabular}


Table 2 continued

\begin{tabular}{|c|c|c|c|c|c|c|c|c|c|c|c|c|c|c|c|c|c|c|c|}
\hline \multirow{2}{*}{ Taxon } & \multicolumn{19}{|c|}{ Character number and states } \\
\hline & 19 & 20 & 21 & 22 & 23 & 24 & 25 & 26 & 27 & 28 & 29 & 30 & 31 & 32 & 33 & 34 & 35 & 36 & 37 \\
\hline Ahtiana aurescens & 1 & 0 & $0 / 2$ & 0 & 0 & 1 & 0 & 2 & 0 & 1 & 0 & 1 & $0 \& 1$ & 0 & 0 & - & 0 & - & 0 \\
\hline A. pallidula & 1 & 0 & 0 & 0 & 0 & 1 & 1 & 2 & 0 & & 0 & 1 & $0 \& 1$ & 0 & & - & 0 & - & 0 \\
\hline A. sphaerosp & 1 & 0 & 0 & 0 & 0 & 1 & $0 \& 1$ & 2 & $0 / 1$ & 1 & 0 & 1 & $0 \& 1$ & 0 & 0 & - & 0 & - & 0 \\
\hline Alloc & 1 & 0 & 0 & 0 & 1 & 1 & $0 \& 1$ & 3 & 2 & 1 & 0 & 1 & & 1 & 0 & - & 0 & - & 0 \\
\hline A. endochr & $?$ & $?$ & $?$ & $?$ & $?$ & 2 & 1 & 3 & 2 & 1 & 0 & 1 & 0 & 1 & 0 & - & 0 & - & 0 \\
\hline A. flavonigrescer & 1 & 0 & $?$ & 0 & 0 & 1 & $0 \& 1$ & 3 & 2 & 1 & 0 & 1 & 3 & 1 & 0 & - & 1 & 0 & 0 \\
\hline A. $g l$ & 1 & 0 & 1 & 0 & 1 & 1 & 0 & 3 & 2 & 1 & 0 & 1 & 0 & 1 & c & - & 0 & - & 0 \\
\hline adre & 1 & 0 & 0 & 1 & 1 & 2 & 1 & 3 & 2 & 1 & 0 & 1 & 0 & 0 & 0 & - & 0 & - & 0 \\
\hline & 1 & 0 & 1 & 0 & 0 & 0 & $0 \& 1$ & 3 & 2 & 1 & 0 & 1 & $0 \& 1$ & 1 & 0 & - & 0 & - & 0 \\
\hline & $?$ & $?$ & $?$ & $?$ & $?$ & 0 & 0 & 3 & & 1 & 0 & 1 & & 0 & 0 & - & 0 & - & 0 \\
\hline & 1 & 0 & 1 & 0 & 0 & 0 & $0 \& 1$ & 3 & 2 & 1 & 0 & 1 & 0 & 1 & 0 & - & 0 & - & 0 \\
\hline Dactylina & 1 & 0 & 0 & 0 & 0 & 2 & 1 & 1 & 0 & 1 & 0 & 0 & - & c & 1 & $1 \& 4$ & 1 & 1 & 0 \\
\hline D. ran & 1 & 0 & 0 & 0 & 0 & 2 & 1 & 1 & 0 & 1 & 0 & ( & - & ( & 1 & 1 & 1 & 1 & 0 \\
\hline & 1 & 0 & 0 & 0 & 1 & 1 & 1 & 2 & 0 & 0 & 1 & 1 & 2 & 1 & 0 & - & 0 & - & 0 \\
\hline "Nep & 1 & 0 & ? & 0 & 1 & 0 & 0 & 2 & 0 & 0 & 0 & 1 & $0 \& 1$ & 0 & 0 & - & 0 & - & 0 \\
\hline Tu & 1 & 0 & 0 & 0 & 0 & 1 & 0 & 2 & 0 & 0 & 1 & 0 & - & 0 & 1 & 0 & 0 & - & 0 \\
\hline bv & 1 & 0 & 0 & 0 & 0 & 0 & 1 & 2 & 0 & 0 & 1 & 1 & 0 & 0 & 0 & - & 0 & - & 0 \\
\hline & 1 & 0 & 0 & 0 & 0 & 1 & 0 & 2 & 0 & 0 & 1 & 0 & - & ( & 1 & $1 \& 2$ & 0 & - & 0 \\
\hline & $?$ & $?$ & $?$ & $?$ & $?$ & $?$ & $?$ & $?$ & $?$ & 0 & 1 & 0 & - & 0 & 1 & 0 & 0 & - & 0 \\
\hline & 1 & 0 & 0 & 0 & 0 & 0 & 1 & 0 & 1 & 0 & 0 & 1 & 0 & ( & 0 & - & 0 & - & 0 \\
\hline & 1 & 0 & $?$ & 0 & 1 & 0 & 0 & 3 & 2 & 0 & 1 & 0 & - & ( & 1 & 3 & 0 & - & 0 \\
\hline & 1 & 0 & 0 & 0 & 0 & 1 & 0 & 2 & 0 & 0 & 0 & 1 & 0 & 0 & 0 & - & 0 & - & 0 \\
\hline & 1 & 0 & 0 & 0 & 0 & 1 & 1 & 2 & 0 & 0 & 1 & 1 & 3 & 1 & 0 & - & 0 & - & 0 \\
\hline Hoi & $?$ & $?$ & $?$ & 0 & 0 & 1 & 0 & 2 & 0 & 0 & 0 & 1 & 0 & 0 & 0 & - & 0 & - & 0 \\
\hline & 1 & 0 & 0 & 0 & 0 & 0 & 0 & 0 & 1 & 0 & 0 & 1 & 0 & 0 & 0 & - & 0 & - & 0 \\
\hline T. ulot & $?$ & $?$ & $?$ & $?$ & $?$ & 0 & 0 & $?$ & $?$ & 0 & 0 & 1 & 0 & 0 & 0 & - & 0 & - & 0 \\
\hline & 1 & 0 & $0 / 2$ & 0 & 0 & 1 & 0 & 2 & 0 & 1 & 0 & 1 & $0 \& 1$ & 0 & 0 & - & 0 & - & 0 \\
\hline & 1 & 0 & 0 & 0 & 0 & 0 & 0 & 2 & 0 & 1 & 0 & 1 & & 0 & 0 & - & 0 & - & 0 \\
\hline T. laxa & 1 & 0 & 0 & 0 & 0 & 0 & 0 & $?$ & $?$ & 1 & 0 & 1 & 0 & 0 & 0 & - & 0 & - & 0 \\
\hline & 1 & 0 & 0 & 0 & 0 & 0 & 0 & 2 & 0 & 1 & 0 & 1 & 0 & 0 & 1 & $0 \& 1$ & 0 & - & 0 \\
\hline T. to & 1 & 0 & 0 & 0 & 0 & 1 & 0 & 2 & 0 & 1 & 0 & 1 & $0 \& 1$ & 0 & 0 & - & 0 & - & 0 \\
\hline Vulpicida can & 0 & 0 & 0 & 0 & 0 & 2 & 1 & 1 & 0 & 1 & 0 & 0 & - & 0 & 0 & - & 0 & - & 1 \\
\hline & 0 & 0 & 0 & 0 & 0 & 1 & 0 & 4 & 1 & 1 & 0 & - & - & 0 & 0 & - & 0 & - & 1 \\
\hline V. pina & 0 & 0 & 0 & 0 & 0 & 1 & 0 & 4 & 1 & 1 & 0 & - & - & 0 & 0 & - & 0 & - & 1 \\
\hline & 0 & 0 & 0 & 0 & 0 & 1 & 0 & 4 & 1 & 1 & 0 & - & - & 0 & 0 & - & 0 & - & 1 \\
\hline & 0 & 0 & 0 & 0 & 0 & 1 & 0 & 4 & 1 & 1 & 0 & 0 & - & 0 & 0 & - & 0 & - & 1 \\
\hline V. viridis & 0 & 0 & 0 & 0 & 0 & $?$ & $?$ & 1 & 0 & 1 & 0 & 0 & - & 0 & 0 & - & $?$ & - & 1 \\
\hline & 0 & 0 & 0 & 1 & 1 & 1 & 1 & 2 & 0 & 0 & 0 & 1 & 0 & 0 & 0 & - & 0 & - & 0 \\
\hline Cetraria islandica & 2 & 1 & 2 & 2 & 1 & 0 & 0 & 1 & 0 & 0 & 0 & 1 & 0 & 0 & 0 & - & 1 & 0 & 0 \\
\hline Flavocetraria & 2 & 1 & 3 & 2 & 1 & 0 & 0 & 2 & 0 & 1 & 0 & 1 & 0 & 0 & 0 & - & 0 & - & 0 \\
\hline Melanelia hepatizon & 0 & 0 & 1 & 2 & & & 0 & & & & 0 & 0 & & 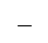 & & - & 1 & 2 & \\
\hline
\end{tabular}




\section{Molecular data}

In the molecular data set, sequences from the ITS1, 5.8S, and ITS2 regions of the nuclear rDNA gene for 23 specimens of 20 cetrarioid lichen species are presented. The matrix contains both sequences newly obtained and those published earlier (Table 3). Seventeen species with globose ascospores from six genera (Ahtiana, Allocetraria, Dactylina, Esslingeriana, Tuckneraria, and Tuckermannopsis) were applied as terminal taxa of the ingroup while three Vulpicida species were used as the outgroup.

The combined length of the sequences obtained from ITS1, 5.8S, and ITS2 was ca 500 nucleotides. In the parsimony analysis of molecular data, 116 informative characters were used.

\section{RESULTS}

\section{Analyses based on morphological data}

Parsimony analysis yielded 7436 equally parsimonious trees (all characters equally weighted, length of trees $=136$ steps, consistency index $\mathrm{CI}=0.382$, homoplasy index $\mathrm{HI}=0.618$, retention index $\mathrm{RI}=0.684$ ). After reweighting the characters by maximum value of rescaled consistency indices 122 equally parsimonious trees (length of trees $=35267$ steps, $\mathrm{CI}=0.677, \mathrm{HI}=0.323$, $\mathrm{RI}=0.882$ ) were obtained. Successive weighting is particularly useful when applied to data sets with much homoplasy (Tehler \& Egea, 1997). The successive approximations character weighting method generated remarkably low weights to some conspicuous morphological characters often used in key-books, such as presence of isidia and soredia (Table 4). This is justified as, in our opinion, these asexual structures are obviously independently derived. In other words, we suppose that sorediate and isidiate species did not share sorediate or isidiate ancestors but came from sexual species instead. The most highly weighted characters are either connected with the anatomy of the thallus (characters $2,3,15$ ), anatomy of ascocarps $(18-22)$, or with secondary compounds of both cortex $(28,29)$ and medulla $(34,36,37)$. Still, some anatomical characters of the ascocarps $(20,22)$ and presence of medullary substances (36) appear to be uninformative in this analysis.

The majority rule consensus trees were obtained in both analyses - with characters equally weighted (Fig. 1) and after the reweighting of characters (Fig. 2). Two major monophyletic groups were found on both trees. Group A consists of 11 species of Tuckermannopsis together with Esslingeriana idahoensis and "Nephromopsis" weii while group B contains eight species of Allocetraria together with two Dactylina species. Furthermore, the clade including five Tuckneraria species (group C) was formed on the majority-rule consensus tree (supported by $95 \%$ of cladograms) with characters equally weighted. Still, this 
Table 3. Sequenced taxa and their GenBank accession numbers (sequences produced for the present paper are in bold)

\begin{tabular}{|c|c|c|c|}
\hline Species & DNA extr. No. & Specimen data & $\begin{array}{c}\text { GenBank } \\
\text { accession No. }\end{array}$ \\
\hline Ahtiana sphaerosporella & DNA\#AT 83 & Canada, British Columbia, Miao \& Taylor (TDI No 211) & AF072224 \\
\hline Allocetraria ambigua & DNA\#AT 874 & China, prov. Sichuan, Obermayer 08141 (GZU) & AF404128 \\
\hline A. flavonigrescens & DNA\#AT 873 & China, prov. Sichuan, Obermayer 08140 (GZU) & AF404127 \\
\hline A. globulans & DNA\#AT 870 & China, prov. Sichuan, Obermayer 08137 (GZU) & AF404126 \\
\hline A. madreporiformis & DNA\#AT 973 & Austria, Tyrol, Obermayer 7746 (M) & AF416460 \\
\hline A. madreporiformis & DNA\#AT 974 & Russia, Krasnoyarsk Terr., Matveeva (M) & AF416461 \\
\hline A. oakesiana & DNA\#AT 136 & Slovenia, Pokorje, Kärnefelt SL-960306 (LD) & AF116179 \\
\hline A. sinensis & DNA\#AT 868 & China, prov. Sichuan, Obermayer 08148 (GZU) & AF404125 \\
\hline A. stracheyi & DNA\#AT 867 & China, prov. Sichuan, Obermayer 08147 (GZU) & AF404124 \\
\hline A. stracheyi & DNA\#AT 875 & China, prov. Sichuan, Obermayer 08139 (GZU) & AF404129 \\
\hline A. stracheyi & DNA\#AT 901 & China, prov. Sichuan, Obermayer 08143 (GZU) & AF404130 \\
\hline Dactylina arctica & DNA\#AT 160 & Canada, Alberta, Miao (TDI No 300) & AF115760 \\
\hline Esslingeriana idahoensis & DNA\#AT 145 & Canada, British Columbia, Goward 961354 (UBC) & AF072227 \\
\hline Tuckermannopsis americana & DNA\#AT 82 & Canada, British Columbia, Miao \& Taylor (TDI No 210) & AF072232 \\
\hline T. chlorophylla & DNA\#AT 525 & Chile, prov. de Magallanes, Feuerer 29475 (TUR) & AF255620 \\
\hline T. platyphylla & DNA\#AT 43 & Canada, British Columbia, Thell \& Veer BC-9643 (LD) & AF072235 \\
\hline T. subalpina & DNA\#AT109 & Canada, British Columbia, Thell BC-9606 (LD) & AF072237 \\
\hline Tuckneraria ahtii & DNA\#AT 607 & Bhutan, Søchting 8489 (LD) & AF404122 \\
\hline T. laureri & DNA\#AT 610 & Bhutan, Wangdi Distr., S $\phi$ chting 8124 (LD) & AF404123 \\
\hline T. pseudocomplicata & DNA\#AT 907 & China, prov. Sichuan, Obermayer $08276 a$ (GZU) & AF404131 \\
\hline Vulpicida canadensis & DNA\#AT 36 & Canada, British Columbia, Thell \& Veer BC-96250 (LD) & AF072238 \\
\hline$V \cdot$ pinastri & DNA\#AT 02 & Canada, British Columbia, Thell SK-9604 (LD) & AF139031 \\
\hline V. tubulosa & DNA\#AT 933 & Austria, Innsbruck, Feuerer \& Thell (HBG) & AF404132 \\
\hline
\end{tabular}


Table 4. Weights of all characters after the second reweighting according to maximum value of $\mathrm{RC}$ indices. Characters with weights over 500 are in bold

\begin{tabular}{|c|c|c|c|}
\hline Character & Inform. & Weight & States \\
\hline 1. Form of thallus & $\mathrm{Y}$ & 111 & 01 \\
\hline 2. Symmetry of thallus & $\mathbf{Y}$ & 1000 & 01 \\
\hline 3. Interior of thallus & $\mathbf{Y}$ & 1000 & 012 \\
\hline 4. Form of lobes & $\mathrm{Y}$ & 91 & 01 \\
\hline 5. Width of lobes & $\mathrm{Y}$ & 42 & 012 \\
\hline 6. Colour of upper surface & $\mathbf{Y}$ & 611 & 012 \\
\hline 7. Colour of lower surface & $\mathbf{Y}$ & 508 & 0123 \\
\hline 8. Pseudocyphellae on upper side & $\mathrm{Y}$ & 0 & 01 \\
\hline 9. Pseudocyphellae on lower side & Y & 175 & 01 \\
\hline 10. Form of pseudocyphellae on lower side & Y & 250 & 01 \\
\hline 11. Cilia & Y & 111 & 01 \\
\hline 12. Rhizines & $\mathrm{Y}$ & 125 & 01 \\
\hline 13. Soredia & Y & 0 & 01 \\
\hline 14. Isidia & $\mathrm{Y}$ & 0 & 01 \\
\hline 15. Structure of cortex & $\mathbf{Y}$ & 1000 & 0123 \\
\hline 16. Cell wall thickness & $\mathrm{Y}$ & 70 & 01 \\
\hline 17. Position of apothecia & Y & 314 & 0123 \\
\hline 18. Ascus shape & $\mathbf{Y}$ & 1000 & 01 \\
\hline 19. Ascus form & $\mathbf{Y}$ & 1000 & 01 \\
\hline 20. Tholus & $\mathbf{N}$ & 1000 & 0 \\
\hline 21. Axial body & $\mathbf{Y}$ & 1000 & 012 \\
\hline 22. Shape of ascospores & $\mathbf{N}$ & 1000 & 01 \\
\hline 23. Length (diameter) of ascospores & $\mathrm{Y}$ & 0 & 01 \\
\hline 24. Position of pycnidia & $\mathrm{Y}$ & 93 & 012 \\
\hline 25. Emergence of pycnidia & $\mathrm{Y}$ & 74 & 01 \\
\hline 26. Shape of pycnoconidia & $\mathbf{Y}$ & 578 & 0123 \\
\hline 27. Length of pycnoconidia & $\mathrm{Y}$ & 308 & 012 \\
\hline 28. Usnic acid & $\mathbf{Y}$ & 1000 & 01 \\
\hline 29. Atranorin & $\mathbf{Y}$ & 1000 & 01 \\
\hline 30. Fatty acids & $\mathrm{Y}$ & 273 & 01 \\
\hline 31. Substance of fatty acids & $\mathrm{Y}$ & 0 & 0123 \\
\hline 32. Secalonic acids & Y & 238 & 01 \\
\hline 33. Orcinol depsides \& depsidones & $\mathrm{Y}$ & 222 & 01 \\
\hline 34. Substance of orcinol depsides \& depsidones & $\mathbf{Y}$ & 1000 & 01234 \\
\hline 35. ß-Orcinol depsidones & $\mathrm{Y}$ & 250 & 01 \\
\hline 36. Substance of B-orcinol depsidones & $\mathbf{N}$ & 1000 & 01 \\
\hline 37. Pulvinic acid derivates & $\mathbf{Y}$ & 1000 & 01 \\
\hline
\end{tabular}




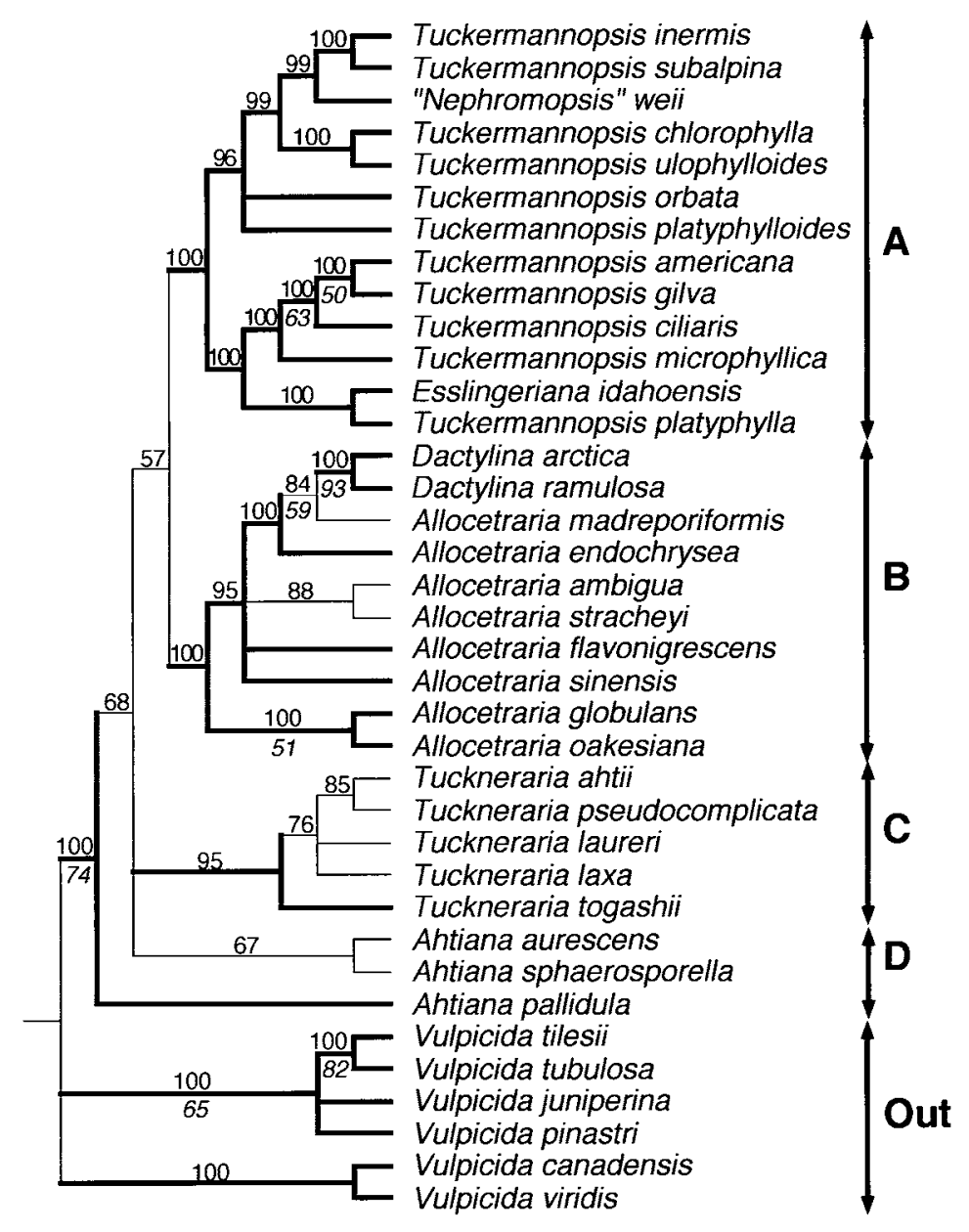

Fig. 1. Majority rule consensus tree of 7436 equally parsimonious cladograms (based on characters with equal weights), obtained as a result of the maximum parsimony analysis of the morphological data set. The length of the shortest trees 136 , consistency index 0.382 , retention index 0.684 . The values above branches correspond to the percentages of equally most parsimonious trees with that specific bipartition; consensus $95 \%$ and more is shown by the thicker branches. Bootstrap values greater than $50 \%$ are shown below branches. Letters A-D indicate informal entities of the ingroup (see the text); Out indicates outgroup taxa.

clade did not reach the level of $50 \%$ on the consensus tree produced after the reweighting of characters. Three species of the genus Ahtiana (group D) did not form a separate clade at all.

Both Bremer support test and bootstrapping were used to find support for the obtained clades. In the Bremer support test, all trees are kept successively one step longer than the shortest tree until all the groups are lost in consensus. 


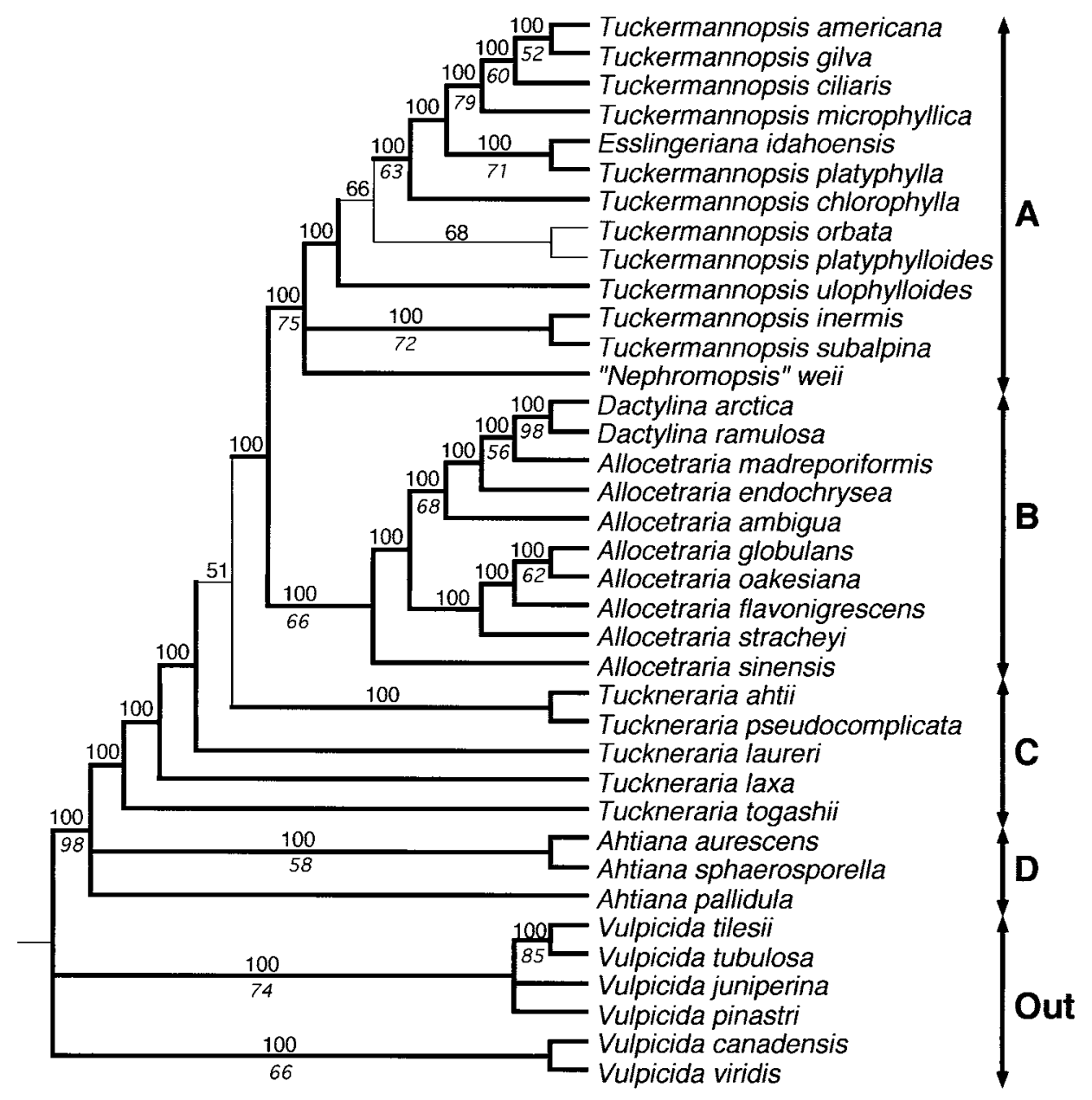

Fig. 2. Majority rule consensus tree of 122 equally parsimonious cladograms (based on reweighted characters), obtained as a result of the maximum parsimony analysis of the morphological data set. The length of the shortest trees 35267 , consistency index 0.677 , retention index 0.882 . The values above branches correspond to the percentages of equally most parsimonious trees with that specific bipartition; consensus $95 \%$ and more is shown by the thicker branches. Bootstrap values greater than $50 \%$ are indicated below branches. Letters A-D mark informal entities of the ingroup (see the text); Out marks outgroup taxa.

Bremer's length difference has also been referred to as the decay test or decay index (Tehler \& Egea, 1997). In our analysis, inclusion of trees one step longer than the shortest tree causes the majority of the tree to collapse into a polytomy and only the Allocetraria-Dactylina clade remains separate. This clade begins to decay at the Bremer support value of three. 
Bootstrap support was not strong for any clades. In the analysis based on characters with equal weights the highest bootstrap values were obtained for the ingroup as a whole (74\%) and two species of Dactylina (93\%) (Fig. 1). Bootstrap values of $70 \%$ and more were obtained in the analysis with reweighted characters only for group A and for four small subclades (Tuckermannopsis microphyllica-T. ciliaris, Esslingeriana idahoensis-Tuckermannopsis platyphylla, Tuckermannopsis inermis-T. subalpina, Dactylina arctica-D. ramulosa) (Fig. 2).

In conclusion, the analyses based on morphological, anatomical, and chemical characters alone were not supported by the decay test or bootstrapping.

\section{Analyses based on molecular data}

The ingroup of the phylogenetic analysis based on the molecular data set included 20 specimens representing 17 species (of the 31 taxa which were morphologically studied). This matrix is composed of complete sequences of the ITS1, 5.8S, and ITS2 genes. Parsimony analysis yielded 20 equally parsimonious trees (length of trees $=411$ steps, consistency index $\mathrm{CI}=0.640$, homoplasy index $\mathrm{HI}=0.360$, retention index $\mathrm{RI}=0.635$ ). Within the ingroup, three monophyletic groups were revealed on the strict consensus tree as well as on the bootstrap 50\% majority-rule consensus tree (Fig. 3). Group A includes a separately branched Tuckermannopsis subalpina and a polytomy consisting of two subclades (the genera Tuckneraria and Tuckermannopsis s. str.) and of three species from different genera (Dactylina arctica, Esslingeriana idahoensis, Tuckermannopsis platyphylla). Group B includes six Allocetraria species (A. ambigua, A. flavonigrescens, A. globulans, two specimens of A. madreporiformis, A. sinensis, and three specimens of $A$. stracheyi) while the seventh taxon from the same genus A. oakesiana - remains unresolved as branch C.

\section{DISCUSSION}

The aim of this phylogenetic analysis was to search for monophyletic groups within the aggregate of cetrarioid lichens characterized by globose ascospores and to verify the correspondence of the current taxonomy to the probable evolution of the taxa involved.

The genus Allocetraria, which at first included only three species from high altitudes in south-east Asia, was introduced by Kurokawa \& Lai (1991). It was separated from Cetraria because of the dichotomously branched lobes, the special appearance of pseudocyphellae, the palisade plectenchymatous cortex, and the unique chemistry. The authors did not pay attention to ascomatal and pycnidial characters. Later studies of these structures confirmed the necessity of a separate genus (Thell et al., 1995c). Today, ten species are combined in the genus; eight 


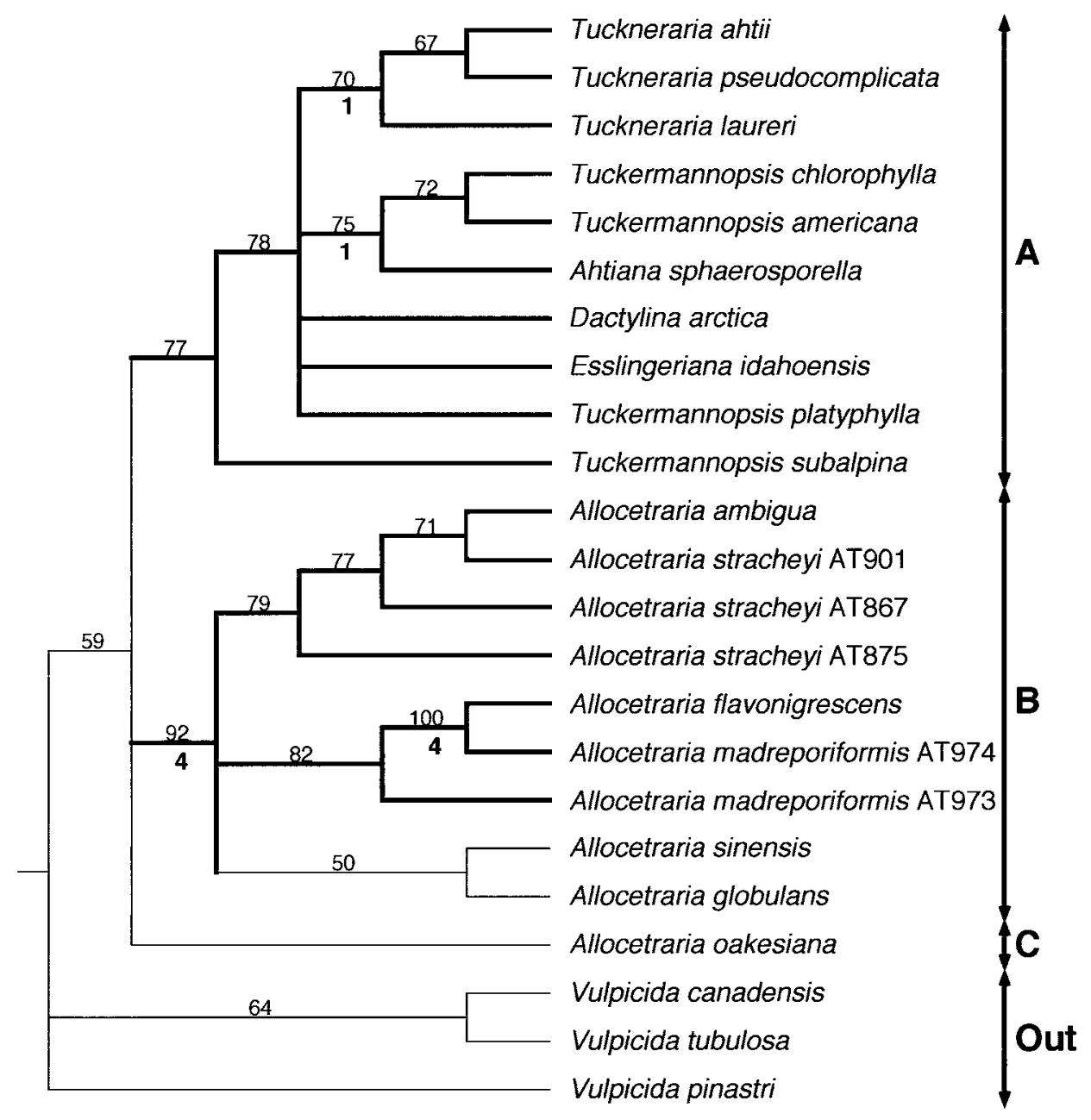

Fig. 3. Bootstrap majority-rule consensus tree, obtained as a result of analysing the molecular data set consisting of 17 species. Bootstrap values $50 \%$ and greater are indicated above branches. Clades supported by bootstrap values $70 \%$ and greater are shown by the thicker lines. Bremer support values are indicated below branches. Letters A-C mark informal entities of the ingroup (see the text); Out marks outgroup taxa.

of them were included in our analyses. We have not seen any herbarium material of A. denticulata, and only one specimen (an isotype material) of A. isidiigera was available. Unfortunately, the descriptions of these two species (Hue, 1899; Kurokawa \& Lai, 1991) are too poor to present them properly in the data matrix. The value of both taxa is rather uncertain - the species are known from the type localities only; and, according to the literature, no fruiting bodies have been observed. 
Six Allocetraria species out of the seven included in our molecular data set form a monophyletic group with high bootstrap and Bremer supports (group B in Fig. 3). The seventh taxon, A. oakesiana, remains separate in this analysis although its morphological, chemical, and anatomical characters show closeness to the other Allocetraria species.

The paraphyletic nature of the genus Allocetraria with respect to two species of Dactylina is assumed on the grounds of morphological characters (Figs. 1, 2). The closeness of these two genera was noticed quite recently, when two former Dactylina species - D. madreporiformis and D. endochrysea - were transferred to Allocetraria (Kärnefelt \& Thell, 1996). The presence of filiform pycnoconidia and asci with an extremely broad axial body is essential for distinguishing Allocetraria. Dactylina is characterized by the radial-symmetrical thallus becoming hollow, the terminal position of apothecia, and also the secondary chemistry. The palisade plectenchymatous arrangement of cortical hyphae, which is rather unusual in the group of cetrarioid lichens, is characteristic of both Allocetraria and Dactylina. Still, the grouping of the species involved into two separate genera is strongly supported by our analysis of molecular characters (Fig. 3) and, therefore, the present nomenclature remains unchanged.

The genus Tuckermannopsis was described by Gyelnik (1933) in a very brief manner: "Affinis generi Nephromopsi sed thallus subtus pseudocyphellis deficientibus". Only two species (T. ciliaris (Ach.) Gyeln. and T. endoxantha (Hue) Gyeln.) were recognized in the genus at that time. T. ciliaris is the type species of the genus while T. endoxantha is not accepted nowadays as its type material was found to comprise two different species, both included in the genus Nephromopsis today (Lai, 1981; Kärnefelt \& Thell, 2001). The genus Tuckermannopsis remained unaccepted for a long time, until the 1980s when Lai (1981) resurrected it. Although the description of the genus was still rather poor, several new combinations were soon proposed (Hale in Egan, 1987; Kurokawa, 1991; Weber in Egan, 1991) to accommodate various cetrarioid species in this genus. Several of those have later been combined into other genera (Ahtiana, Allocetraria, Kaernefeltia, Melanelia, Vulpicida). After numerous rearrangements eleven species are still recognized in Tuckermannopsis (Randlane et al., 1997; Randlane \& Saag, 1998). At present it is generally accepted that globose ascospores in narrowly clavate asci, large axial body, dumb-bell shaped pycnoconidia, and moderately small foliose brown to greenish thallus (indicating the absence of usnic acid in the cortex) are the important characters in delimiting the genus. The defining character proposed by Gyelnik - presence of pseudocyphellae on the lower surface of thallus - is not considered to be of importance any longer. A recent review of the genus (Kärnefelt \& Thell, 2001) indicates that only seven species (T. americana, T. chlorophylla, T. ciliaris, T. gilva, T. microphyllica, T. orbata, and T. ulophylloides) should be included in the genus s. str. while the positions of T. inermis, T. platyphylla, T. platyphylloides, and T. subalpina are doubtful. Still, molecular data are available for four 
Tuckermannopsis species (T. americana, T. chlorophylla, T. platyphylla, and T. subalpina) only.

According to our phylogenetic analysis based on morphological characters (Figs. 1,2), the genus Tuckermannopsis, as it is generally accepted (including eleven species), should be considered paraphyletic. Esslingeriana idahoensis, the sole species of the genus Esslingeriana, is predominantly connected with Tuckermannopsis platyphylla and this pair of species always branches out next to the so-called Tuckermannopsis ciliaris group. Monophyletic origin can be declared only for this group. The subclade consisting of four apparently closely related species (T. americana, T. ciliaris, T. gilva, T. microphyllica) is also supported by bootstrapping (bootstrap value $79 \%$ ).

"Nephromopsis" weii, recently described from China (Chen \& Gao 2001), is another taxon which is included in the clade mainly consisting of Tuckermannopsis species (group A in Figs. 1 and 2). There is no doubt that this lichen does not belong to the genus Nephromopsis, which is characterized by rather large foliose thalli of yellowish colour (containing usnic acid), the presence of pseudocyphellae only on the lower surface, marginal apothecia situated on the lower side, and narrowly clavate asci with ellipsoid ascospores. "Nephromopsis" weii has olive brown thallus, pseudocyphellae present on both surfaces, and ascospores clearly globose (6-7 $\mu \mathrm{m}$ in diameter) (Chen \& Gao, 2001). A new combination for this taxon can be presented after additional studies have been carried out on the type specimen.

The phylogenetic analysis based on molecular characters includes only four Tuckermannopsis species out of eleven. It reveals the closeness of T. americana and $T$. chlorophylla while $T$. subalpina branches out separately and the position of T. platyphylla remains unresolved in a polytomy together with Dactylina arctica and Esslingeriana idahoensis (Fig. 3). In our opinion, any further taxonomical rearrangements in this genus would not be justified until additional molecular research has been carried out.

The recently proposed genus Tuckneraria (Randlane et al., 1994) includes five species, which predominantly grow in eastern and south-eastern Asia. They were transferred mainly from Nephromopsis to the new genus because of important anatomical characters (globose ascospores and asci of the same form as in Tuckermannopsis). Later the idea of close affinities between Tuckneraria and Ahtiana was proposed (Thell et al., 1995a). The monophyletic origin of the genus Tuckneraria is supported in one series of current analyses based on the morphological data set (with equally weighted characters) (group C in Fig. 1); in the other series (after the reweighting of characters), the species of Tuckneraria do not form a separate clade but branch out successively (group C in Fig. 2). Phylogenetic analysis based on molecular characters exhibits the monophyletic origin of all three Tuckneraria species (T. ahtii, T. laureri, and T. pseudocomplicata) included in the analysis (Fig. 3).

The originally monotypic genus Ahtiana was segregated from Parmelia s. lat. on the basis of emergent pycnidia, globose ascospores, leptodermatous cortex, 
and the presence of medullary caperatic acid (Goward, 1985). Despite its parmelioid habit with laminal apothecia and pycnidia, Ahtiana sphaerosporella was shown to be closely allied to Cetraria pallidula (Goward, 1985). Today the genus includes three species (Thell et al., 1995a). In our present analysis based on morphology, two species, A. sphaerosporella and A. aurescens, form a pair while A. pallidula branches out separately (group D in Figs. 1 and 2). The analysis based on ITS sequences indicates a close relationship between the genera Ahtiana (represented by the type species A. sphaerosporella) and Tuckermannopsis (Fig. 3) while Tuckneraria species form a separate subclade.

\section{ACKNOWLEDGEMENTS}

This research was made possible by grant No. 3921 from the Estonian Science Foundation. Austrian Science Fund (P1376-BIO) financed the expedition to Tibet in the summer of 2000, during which the fourth author collected numerous herbarium specimens used for the present analysis. The DNA studies (carried out by the third author) were supported financially by grant No. 44079 from the Academy of Finland.

We are greatly indebted to Erast Parmasto and Anders Tehler for comments on the manuscript, and Tassilo Feuerer for general support. Triin Randlane is thanked for revising the English text.

\section{REFERENCES}

Chen, L. \& Gao, X. 2001. Two new species of Nephromopsis (Parmeliaceae, Ascomycota). Mycotaxon, 77, 491-496.

Crespo, A. \& Cubero, O. 1998. A molecular approach to the circumscription and evaluation of some genera segregated from Parmelia s. lat. Lichenologist, 30, 369-380.

Crespo, A., Gavilán, R., Elix, J. \& Gutiérrez, G. 1999. A comparison of morphological, chemical and molecular characters in some parmelioid genera. Lichenologist, 31, 451-460.

Culberson, C. F. 1972. Improved conditions and new data for the identification of lichen products by a standardized thin-layer chromatographic method. J. Chromatogr., 72, 123-125.

Culberson, C. F., Culberson, W. L. \& Johnson, A. 1981. A standardized TLC analysis of B-orcinol depsidones. Bryologist, 84, 16-29.

Egan, R. S. 1987. A fifth checklist of the lichen-forming, lichenicolous and allied fungi of the continental United States and Canada. Bryologist, 90, 77-173.

Egan, R. S. 1991. Changes to the "Fifth checklist of the lichen-forming, lichenicolous and allied fungi of the continental United States and Canada". Bryologist, 94, 396-400.

Esslinger, T. 1971. Cetraria idahoensis, a new species of lichen endemic to western North America. Bryologist, 74, 364-369.

Gardes, B. \& Bruns, T. D. 1993. ITS primers with enhanced specificity for basidiomycetes application to the identification of mycorrhizae and rusts. Molec. Ecol., 2, 113-118.

Gilbert, D. 1993. SeqApp, a Biological Sequence Editor and Analysis Program. Indiana Univ., Bloomington.

Goward, T. 1985. Ahtiana, a new lichen genus in the Parmeliaceae. Bryologist, 88, 367-371. 
Gyelnik, V. 1933. Lichenes varii novi criticique. Acta Fauna Fl. Universali, Ser. 2, 1 (5-6), 3-10.

Huang, X. 1992. A contig assembly program on sensitive detection of fragment overlaps. Genomics, $14,18-25$.

Hue, A.-M. 1899. Lichenes extra-europaei (Suite). Nouv. Arch. Mus. Hist. Nat., Ser. 4, 1, 27-220.

Kärnefelt, I. \& Thell, A. 1996. A new classification for the Dactylina/Dufourea complex. Nova Hedwigia, 91, 595-605.

Kärnefelt, E. I. \& Thell, A. 2000. On the systematic position of the genus Cetrariella (Parmeliaceae: Ascomycotina) indicated by ITS rDNA data. In New Aspects in Cryptogamic Research. Contributions in Honour of Ludger Kappen (Schroeter, B., Schlensog, M. \& Green, T. G. A., eds.). Biblioth. Lichenol., 75, 27-32.

Kärnefelt, E. I. \& Thell, A. 2001. Delimitation of the lichen genus Tuckermannopsis Gyeln. (Ascomycotina: Parmeliaceae) based on morphology and DNA sequences. In Lichenological Contributions in Honour of Jack Elix (McCarthy, P. M., Kantvilas, G. \& Louwhoff, S. H. J. J., eds.). Biblioth. Lichenol., 78, 193-209.

Kärnefelt, I., Mattsson, J.-E. \& Thell, A. 1992. Evolution and phylogeny of cetrarioid lichens. Pl. Syst. Evol., 183, 113-160.

Kurokawa, S. 1991. Japanese species and genera of the Parmeliaceae. J. Jap. Bot., 66, 152-159.

Kurokawa, S. \& Lai, M.-J. 1991. Allocetraria, a new genus in the Parmeliaceae. Bull. Natl. Sci. Mus. (Tokyo), B, 17, 59-65.

Lai, M.-J. 1981 [1980]. Studies on the cetrarioid lichens in Parmeliaceae of East Asia. I. Quart. J. Taiwan Mus., 33, 215-229.

Mattsson, J.-E. 1993. A monograph of the genus Vulpicida (Parmeliaceae, Ascomycetes). Opera Bot., 119, 1-61.

Mattsson, J.-E. \& Wedin, M. 1998. Phylogeny of the Parmeliaceae - DNA data versus morphological data. Lichenologist, 30, 463-472.

Myllys, L., Lohtander, K., Källersjö, M. \& Tehler, A. 1999. Sequence insertions and ITS data provide congruent information on Roccella canariensis and $R$. tuberculata (Arthoniales, Euascomycetes) phylogeny. Molec. Phylog. Evol., 12, 295-309.

Randlane, T. \& Saag, A. 1998. Changes in systematics of cetrarioid lichens. Sauteria, 9, 43-50.

Randlane, T. \& Saag, A. 2000. Revision of the second updated world list of cetrarioid lichens. Online. Internet, 16. August 2000 - http://www.ut.ee/lichens/cetraria.html

Randlane, T., Saag, A. \& Thell, A. 1997. A second updated world list of cetrarioid lichens. Bryologist, 100, 109-122.

Randlane, T., Saag, A., Thell, A. \& Kärnefelt, I. 1994. The lichen genus Tuckneraria Randlane \& Thell - a new segregate in the Parmeliaceae. Acta Bot. Fenn., 150, 143-151.

Saag, A. \& Randlane, T. 1995. Phylogenetic affinities of cetrarioid lichens. Cryptog. Bot., 5, $128-136$.

Swofford, D. L. 1993. PAUP: Phylogenetic analysis using parsimony, version 3.1.1. Computer program distributed by the Illinois Natural History Survey.

Tehler, A. \& Egea, J. M. 1997. The phylogeny of Lecanactis (Opegraphaceae). Lichenologist, 29, 397-414.

Thell, A. 1996. Anatomy and taxonomy of cetrarioid lichens. Summary of doctoral dissertation. Department of Systematic Botany, Lund University.

Thell, A. 1998. Phylogenetic relationships of some cetrarioid species in British Columbia with notes on Tuckermannopsis. Folia Cryptog. Estonica, 32, 113-122.

Thell, A. 1999. Group I introns versus ITS sequences in phylogeny of cetrarioid lichens. Lichenologist, 31, 441-449.

Thell, A. \& Miao, V. 1998. Phylogenetic analysis of ITS and group I intron sequences from European and North American samples of cetrarioid lichens. Ann. Bot. Fenn., 35, 275-286.

Thell, A., Goward, T., Randlane, T., Kärnefelt, E. I. \& Saag, A. 1995a. A revision of the North American lichen genus Ahtiana (Parmeliaceae). Bryologist, 98, 596-605. 
Thell, A., Mattsson, J.-E. \& Kärnefelt, I. 1995b. Lecanoralean ascus types in the lichenized families Alectoriaceae and Parmeliaceae. Cryptog. Bot., 5, 120-127.

Thell, A., Randlane, T., Kärnefelt, T., Gao, X.-Q. \& Saag, A. 1995c. The lichen genus Allocetraria (Ascomycotina, Parmeliaceae). In Flechten Follmann. Contributions to Lichenology in Honour of Gerhard Follmann (Daniels, F. J., Schulz, M. \& Peine, J., eds.), pp. 353-370. University of Cologne, Germany.

Thell, A., Berbee, M. \& Miao, V. 1998. Phylogeny of the genus Platismatia based on rDNA ITS sequences (lichenized Ascomycotina). Cryptog., Bryol. Lichénol., 19, 43-54.

Thell, A., Stenroos, S. \& Myllys, L. 2000. A DNA study of the Cetraria aculeata and C. islandica group. Folia Cryptog. Estonica, 36, 95-106.

Wedin, M., Döring, H. \& Mattsson, J.-E. 1999. A multi-gene study of the phylogenetic relationships of the Parmeliaceae. Mycol. Res., 103, 1185-1192.

White, T. J., Burns, T., Lee, S. \& Taylor, J. 1990. Amplification and direct sequencing of fungal ribosomal DNA genes for phylogenetics. In PCR Protocols: A Guide to Methods and Applications (Innis, M., Gelfand, J., Sninsky, J. \& White, T., eds.), pp. 315-322. Academic Press, Orlando.

\section{Ümmarguste kotteostega tsetrarioidsete samblike fülogeneetiline analüüs}

\section{Andres Saag, Tiina Randlane, Arne Thell ja Walter Obermayer}

Ümmarguste kotteostega tsetrarioidsete samblike rühma kuulub 37 liiki kaheksast perekonnast. Arvutiprogrammi PAUP 3.1.1 abil tehti selle rühma fülogeneetiline analüüs kasutades kahte andmemaatriksit - morfoloogilisi (sh anatoomilisi ja keemilisi) tunnuseid sisaldavat maatriksit ning molekulaarseid tunnuseid (ITS1, 5.8S ja ITS2 rDNA nukleotiidide järjestusi) sisaldavat maatriksit - eesmärgiga kontrollida praegu kasutusel oleva süsteemi vastavust nimetatud taksonite võimalikule evolutsioonile. Morfoloogiliste tunnuste põhjal võib oletada, et perekond Allocetraria on parafüleetiline kahe Dactylina liigi suhtes. Nimetatud perekondade eristamist toetavad tugevalt siiski molekulaarandmed. Perekond Tuckermannopsis laias mahus (sisaldab 11 liiki) on parafüleetiline liikide Esslingeriana idahoensis ja "Nephromopsis" weii suhtes. Morfoloogiliste tunnuste põhjal saab selles klaadis monofüleetiliseks pidada üksnes perekonnasisest mitteformaalset rühma, mis sisaldab liiki Tuckermannopsis ciliaris ja talle lähedasi taksoneid. Molekulaarsed tunnused viitavad perekonna Tuckneraria kolme uuritud liigi monofüleetilisusele. Oletus perekonna Ahtiana ühtsest päritolust ei leia morfoloogiliste andmete põhjal toetust. 